\title{
Comparison of Painful Response to Mechanical Stimulation of the Plantar and Dorsal Surface of Paw Following Chronic Constriction Injury-induced Neuropathic Pain
}

\author{
Ali Ghanbari ${ }^{1^{*}}$ and Mohadeseh Mohammadi ${ }^{2}$ \\ ${ }^{1}$ Research Center of Physiology, Semnan University of Medical Sciences, Semnan, Postal Cod: 351989995, Iran \\ ${ }^{2}$ Student Research Committee, Semnan University of Medical Sciences, Semnan, Postal Cod: 351989995, Iran \\ *Corresponding author's Email: ghanbari@semums.ac.ir; (DORCiD: 0000-0002-7568-3681
}

\begin{abstract}
Mechanical and thermal stimuli were used to evaluate neuropathic pain-like behavior in animal models usually. Mechanical stimulation of paw plantar surface is commonly used to determine mechanical allodynia. In the present study, paw withdrawal response to plantar surface stimulation was compared with paw withdrawal response to dorsal surface stimulation. To this end, a total of 30 female Wistar rats (180-220 g), were assigned randomly to three groups as intact (without any manipulation), sham (incision of skin and muscles without nerve injury), and neuropathy (sciatic nerve lesion) with 10 in each group. To induction of neuropathy (chronic constriction injury), four movable ligations were established around the sciatic nerve using catgut chromic suture with a distance of one millimeter apart and then wound incision was closed. In the sham group, the incision site was closed without nerve ligation. Mechanical allodynia was examined by Von Frey filaments for four weeks. The findings indicated that the paw withdrawal threshold following dorsal surface stimulation was significantly reduced compared to the sham group at day 21 post-surgery. Moreover, paw withdrawal threshold following plantar surface stimulation significantly decreased compared to the sham group at day 21 post-surgery. The present results regarding the sham group showed that the paw withdrawal threshold after mechanical stimulation of the plantar surface was not significantly different from that of the dorsal surface paw. In addition, and there was no significant difference between the paw withdrawal response to plantar surface and dorsal one. In conclusion, paw withdrawal threshold to plantar surface mechanical stimulation was not significantly different from one in dorsal surface following neuropathic pain induced by chronic constriction injury.
\end{abstract}

Keywords: Mechanical allodynia, Neuropathic pain, Paw dorsal surface, Paw plantar surface, Rat

\section{INTRODUCTION}

Neuropathic pain is chronic pain, caused as a result of a lesion or disease of the central or peripheral nervous system. Spontaneous pain, allodynia (pain sensation following non-noxious stimuli), and hyperalgesia (exaggerated pain following noxious stimuli) are some of its signs (Ji and Suter, 2007; Popiolek-Barczyk and Mika, 2016; Carrasco et al., 2018). Despite several studies toward neuropathic pain treatment, no successful treatment has been presented so far (Kingery, 1997; Chanchal et al., 2016). Due to the ethical issues and the impossibility of creating a human model of pain, most of the available information is the result of research on animal models (Mogil, 2009; Deng et al., 2021), and a few available human studies have addressed people who have suffered from the pain caused by traumatic events. Since pain is a subjective matter and animals are unable to express it, the animal's response to the stimulus with behaviors, such as withdrawing the limb from the stimulus, does not necessarily mean pain-like behavior (Deuis et al., 2017), therefore, various methods have been introduced to evaluate pain-like behavior in animals, each of which has some advantages and disadvantages. However, there is a need to consider the benefits and drawbacks of each method to improve our understanding of pain.

There are different methods to determine pain-like responses in animals, including putting foot or tail in hot water, infrared radiation to paw plantar surface or tail, putting the animal on a hot plate device, clamping the skin with a certain amount of force, stimulating the plantar or dorsal surface of the paw or cheeks of the animal with polyethylene filaments, and applying pressure or pushing a sharp point object on the skin (Jensen and Finnerup, 2014; Deuis et al., 2017). Among the mentioned methods, mechanical stimulation of the paw plantar surface with polyethylene filaments is one of the most common methods used to evaluate mechanical pain in animals (Yam et al., 2020). In this method, the animal is placed in a cage with meshed metal floor and the researcher triggers the pain response by applying the Von Frey filament to the plantar surface of the paw (Jensen and Finnerup, 2014) which the animal responds by lifting and moving its foot away from the stimulus.

Recently, researchers have also used the dorsal surface of the foot to examine the response to mechanical stimulation. In each case, the animal is placed on a table and the researcher holds the animal with one hand and places 
the filament on the animal's foot (the skin between the toes 2 and 3) to trigger a pain-like response by considering raising the leg as the criterion for pain (Ren, 1999; Safakhah et al., 2017; Pop et al., 2021). According to common methods, the plantar surface but not the dorsal surface, is used to determine thermal pain that may be different from the dorsal surface in point of withdrawal response to mechanical stimulus. Therefore, it is necessary to clarify whether the plantar surface response is the same as the dorsal surface response. Therefore, due to the fact that in some studies, the plantar surface of the foot is used to evaluate mechanical allodynia, and in others, the dorsal surface of the foot is used, so in the present study, the pain-like response of paw plantar surface was compared with that of dorsal surface in the chronic constriction injury-induced neuropathic pain in rats.

\section{MATERIALS AND METHODS}

\section{Ethical approval}

The present study was approved by the Ethics Committee of the Faculty of Medicine, Semnan University of Medical Sciences, Iran (Certificate Number: IR.SEMUMS. REC.1398.162). All experiments were performed in accordance with National Institutes of Health guidelines for working with laboratory animals. To minimize diurnal variations, all experiments were carried out between 2 and 5 PM.

\section{Animals}

In the present study, female Wistar rats, weighing $200 \pm 20 \mathrm{~g}$ were housed in a place with controlled temperature $\left(22 \pm 2{ }^{\circ} \mathrm{C}\right)$, and 12 hours light-dark cycles were used. The rat fed and watered ad libitum. A total of 30 rats were divided into three groups of intact, sham, and neuropathy with 10 in each group. It should be mentioned that animals in the intact group did not receive the intervention (for comparison with the sham group to show the effect of incision on pain-like behavior). In the sham group (as the control group for neuropathy) only skin and muscles were incised while in the neuropathy [chronic constriction injury $(\mathrm{CCI})$ ] group, skin and muscles at the sciatic nerve were incised and sciatic nerve was sutured.

\section{Neuropathic pain induction}

Chronic constriction injury of the sciatic nerve was made using the method described by Bennett and Xie (1988). Animals were anesthetized using intraperitoneal injection of a mixture of ketamine hydrochloride and xylazine ( 80 and $10 \mathrm{mg} / \mathrm{kg}$, respectively, Vafaei et al., 2020) and the upper right thigh was shaved and then a $2 \mathrm{~cm}$ incision was made in the place of the sciatic nerve. The sciatic nerve was exposed and separated from surrounding tissues. The nerve was ligated using four catgut chromic sutures $4 / 0$, with a $1 \mathrm{~mm}$ distance between each and then the incision site was closed using silk suture 4/0. Animals in the sham group received surgery without nerve ligation. The rats were housed in individual cages until full consciousness was regained.

\section{Evaluation of mechanical pain-like behavior}

Mechanical allodynia was evaluated using Von-Frey filaments in the plantar and dorsal surface of the foot using methods described by Ren (1999), and Bennett and Xie (1988), respectively. Von-Frey filament is a polyethylene hair, which is calibrated according to its diameter. Each filament applies a certain amount of force to the surface on which it is pressed. The filaments are used increasingly from low strength toward stronger ones. Each filament was used 5 times with 10 seconds interval between each stimulation. If the animal responded to three consecutive stimulations, this force would be considered as the response threshold; and otherwise, the stimulation would be continued with the stronger filament. The experiments were performed first with the plantar surface as described by Bennett and Xie (1998) and then continued one hour later with the dorsal surface following the method proposed by Ren (1999). Pain-like behavior assessments were performed at the end of the first, second, third, and fourth weeks post-surgery. A force of $60 \mathrm{~g}$ was considered as the cutoff point.

\section{Statistical Analysis}

All data were analyzed using GraphPad Prism version 8.0 software (GraphPad, San Diego, CA, USA). Regarding the normal distribution of data (using the Kolmogorov-Smirnov test), two-way analysis of variance (ANOVA) and then Sidak's multiple comparisons test were used. All data were expressed as mean \pm SEM of the examined variable. $\mathrm{P}<0.05$ was considered statistically significant.

\section{RESULTS}

The obtained result showed that there was no significant difference between the intact and sham groups regarding the pain-like response to mechanical stimulation of the plantar and dorsal surfaces of the paw ( $\mathrm{p}>0.05)$. Moreover, the 
withdrawal threshold following plantar surface stimulation was similar to the response of dorsal surface in both intact and sham groups (data not shown).

The current findings indicated that chronic constriction injury (CCI) significantly reduced paw withdrawal threshold following dorsal surface stimulation, compared to the sham group. The findings indicated that the paw withdrawal threshold significantly decreased during 21 days post-surgery $(\mathrm{p}<0.05)$ and increased on day 28 in the CCI group, compared to the sham group ( $\mathrm{p}<0.05$, Figure 1). Regarding plantar surface, the present results showed that paw withdrawal threshold following plantar surface stimulation significantly reduced in the CCI group during day 21 postsurgery, compared to the sham group, and continued to decrease until day 28 post-surgery ( $<<0.05$, Figure 2). Paw withdrawal threshold following mechanical stimulation of the plantar surface was not significantly different from that of the dorsal surface in the sham group (Figure 3).

In addition, in the sciatic nerve lesion group (CCI), there was no significant difference between the withdrawal responses induced by the mechanical stimulation of the plantar surface, compared to the dorsal surface response (Figure 4). Withdrawal response diagram following mechanical stimulation of the paw plantar and dorsal surface showed a downward trend from the seventh day following the injury and continued until the end of the experiment.

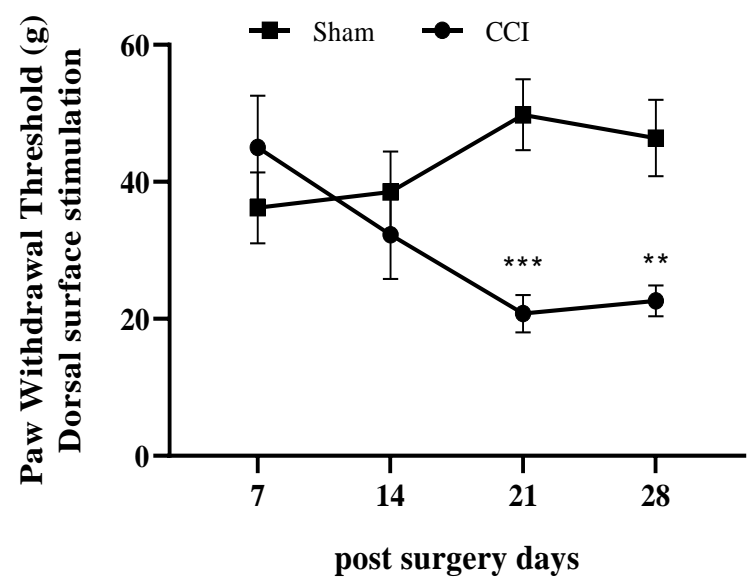

Figure 1. Withdrawal threshold following mechanical stimulation of the dorsal surface of the paw. Withdrawal response following mechanical stimulation of dorsal paw significantly reduced at third weeks in the neuropathy group, compared to the sham group, and continued to the end of the experiment (fourth week). Data are expressed as mean \pm SEM with 10 rats in each group. ${ }^{* *} \mathrm{p}<0.01, * * * \mathrm{p}<0.001$

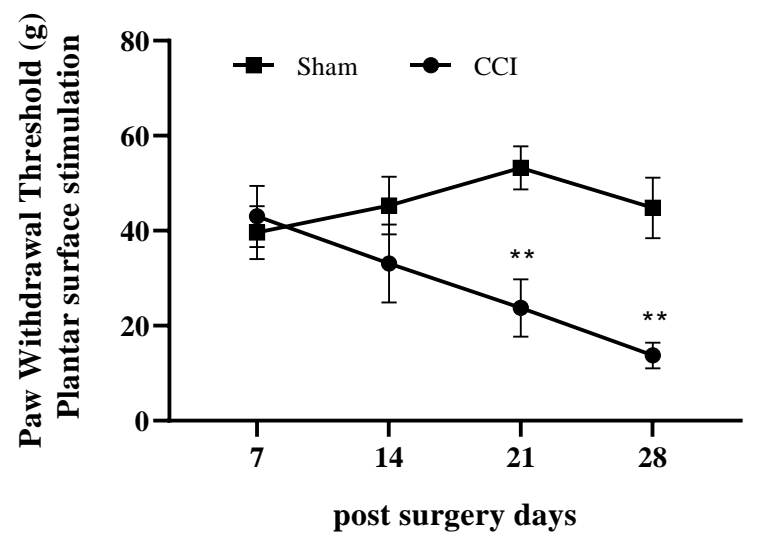

Figure 2. Withdrawal threshold following mechanical stimulation of the plantar surface of the paw. Withdrawal response following mechanical stimulation of paw plantar surface significantly reduced at third weeks in the neuropathy group, compared to the sham group and continued to the end of the experiment (fourth week). Data are expressed as mean \pm S.E.M. with 10 rats in each group. $* * \mathrm{p}<0.01$

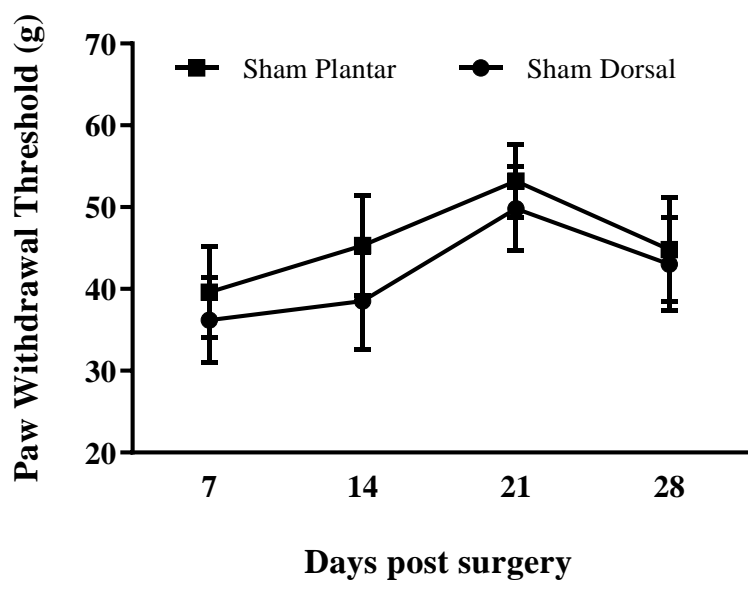

Figure 3. Comparison of withdrawal response threshold following mechanical stimulation of the plantar surface and dorsal surface of the foot in the sham group. Paw withdrawal threshold following mechanical stimulation of the plantar surface did not show a significant difference, compared to the dorsal surface in the sham group. Data are expressed as mean \pm SEM with 10 rats in each group.

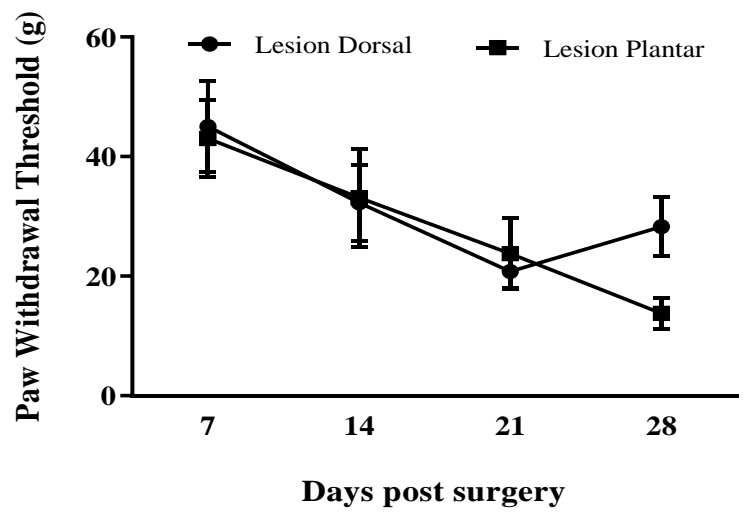

Figure 4. Comparison of withdrawal response threshold following mechanical stimulation of the plantar surface and dorsal surface of foot in the chronic constriction injuryinduced neuropathy group. Paw withdrawal threshold following mechanical stimulation of the plantar surface did not show a significant difference compared to the dorsal surface in the neuropathy group. Withdrawal response diagram following mechanical stimulation of plantar and dorsal surface showed a downward trend from the seventh day following the injury and continued until the end of the experiment. Data are expressed as mean \pm SEM with 10 rats in each group. 
In the present study, the responses to mechanical stimulation of the plantar surface and dorsal surface of the foot following chronic constriction injury of the sciatic nerve were evaluated.

Comparison of animals' painful responses following mechanical stimulation of the paw plantar surface and dorsal surface in intact group and sham group showed that incision of skin and tissue around the nerve had no significant effect on the response to mechanical stimulation.

According to the present results, there was no significant difference between the withdrawal response of the plantar surface and dorsal surface due to mechanical stimulation of the plantar and dorsal surfaces following sciatic nerve injury. Several studies have been performed on different methods of determining pain in laboratory animals (Taiwo et al., 1989; Santos-Nogueira et al., 2012; Jensen and Finnerup, 2014; Deuis et al., 2017), however, there is no report about possible difference between the pain-like response due to plantar surface stimulation and dorsal surface stimulation in CCIinduced neuropathic pain rats.

The results of the current experiments showed that the withdrawal threshold following plantar surface stimulation was significantly different from the control group on day 21 after CCI, which was similar to the dorsal surface response. This result was in agreement with previous results indicating that two to three weeks after CCI surgery, the response threshold to mechanical stimulation significantly reduced, compared to the control group (Safakhah et al., 2016; Safakhah et al., 2017). The present results were also consistent with studies that showed two to three weeks after spinal cord injury; the paw withdrawal threshold significantly decreased, compared to the control group (Masri et al., 2009). Withdrawal response during plantar surface mechanical stimulation was not significantly different compared to dorsal surface stimulation in both the sham group and CCI group. This result was inconsistent with the results of Soignier et al (2011) indicating complete Freund's adjuvant (CFA)-induced pain which showed withdrawal threshold following dorsal surface stimulation was higher than the plantar surface threshold (Soignier et al., 2011). However, there were differences between the findings of the current study and those reported by Soignier et al (2011). Unlike neuropathic pain that was evaluated in the current study, Soignier et al (2011) assessed nociceptive pain. Furthermore, the current study examined pain-like behavior over a long period of time after the injury (at least 1 week), while Soignier et al (2011) evaluated pain behavior for a few hours following CFA administration. Finally, the method of pain induction in the current study was different from the method used by Soignier et al (2011).

Although there was no difference between the pain-like response following stimulation of the plantar surface and the dorsal surface of the foot, the determination of the pain-like behavior with the plantar surface method was associated with problems suggesting that the dorsal surface method was preferable. Pitcher et al. (1999) reported that the surface on which the animal stand can affect the animals' response by affecting sensory processing. They showed that if the animals' foot is on a wire mesh surface, determining the pain-like response from the plantar surface is associated with a lot of variation, whereas if a Plexiglas surface is used, this variation will be reduced. Given that in most cases, to assess the mechanical pain-like response, the animal is placed in cages with a wire mesh floor leading to a variety of responses, and then ambiguous and incorrect interpretations. Moreover, it has been reported that wire mesh floor possibly leads to tactile hyperstasis (Mizisin et al., 1998) which in turn affects the data.

It has also been reported that weight-bearing, which can be considered as pressure on the plantar surface of the foot, can be a confounding factor in assessing the pain-like response through the plantar surface (Kauppila et al., 1998).

According to the above reports, withdrawal response may be due to plantar surface stimulation could not be a result of von-Frey filament stimulation entirely, so part of which possibly is a result of tactile hyperstasia due to wire mesh floor contact. Possibly, discomfort due to the weight-bearing of the hind paws that are placed on the wire mesh can cause a change in sensory processing and create a non-pain response and thus mislead evaluating the pain-like response (Kauppila et al., 1998). Therefore, the observed reaction following the stimulation of the plantar surface of the foot is somewhat suspicious in comparison with dorsal surface stimulation. The following reasons, which are disadvantages of the plantar stimulation method for determining pain-like behavior, can further clarify the issue.

First, detection of withdrawal response through plantar surface stimulation takes a long time to stabilize the animal (in mice, it may take up to an hour, Minett et al., 2011), and therefore prolonged contact of the plantar surface of the foot with the wire mesh causes the animal to move. Second, the desired area for detecting withdrawal response may be covered by the wire mesh and thus the determination of pain from the same area may not be recorded in all animals (Pitcher et al., 1999). Third, moving the foot after one stimulation may cause the different area to be accessible and so the stimulation of the next time at the previous area is impossible and therefore creates a different response. In this regard, it has been reported that the thickness of the epidermis and CGRP-releasing fibers in the medial and lateral regions of the plantar surface are different from each other, and especially that surgical intervention increases the effect of filaments from the medial to the lateral (Duraku et al., 2012) which can lead to a different response. Moreover, there was a need to contact the filament perpendicular to the surface of the plantar surface of the animals' foot, so insufficient visual control of the desired position makes it impossible to stimulate the same area in all cases. In addition, the filament 
may come in contact with the plantar surface of the foot suddenly or diagonally, which in both cases can cause scratches, and therefore the animal moves its foot away from the stimulus and this movement may consider as a pain-like response. Moreover, it is possible that repetitive stimuli may cause the animal to learn to move its foot away to prevent further stimulation and the inexperienced researcher may mistakenly consider this answer as a pain-like behavior. The fifth reason is that, in this method, a strong filament can move (raise) the foot and the researcher considers the observed response to be a pain-like behavior in the animal (Tal and Bennett, 1994; Xiao and Bennett, 1994; Ren et al., 1995). On the other hand, in the dorsal surface stimulation method, the withdrawal response following the stimulation is quite obvious and creates more confidence in the observed response as a pain-like reaction. This idea is consistent with Soignier et al (2011), who stated that the stimulation on the dorsal surface shows a much more accurate correct painful response (Soignier et al., 2011). According to the above-mentioned points, determining the pain-like behavior following stimulation of the dorsal surface can give a more obvious and acceptable criterion than stimulation of the plantar surface of the foot and increase the validity of the obtained data.

\section{CONCLUSION}

The pain-like response due to mechanical stimulation of the plantar surface of the foot is not significantly different from the response due to the stimulation of the dorsal surface, but assessing the pain-like response from the dorsal surface of the foot is easier and more reliable than the method of determining the response from the plantar surface.

\section{DECLARATIONS}

\section{Authors' contribution}

Ali Ghanbari designed the overall study and wrote the paper. Ali Ghanbari and Mohadeseh Mohammadi performed the experiment, collected, and processed the data. All authors discussed the results and commented on the manuscript. The final draft of the manuscript and statistical analysis were confirmed by the authors.

\section{Conflict of interests}

The authors declare no conflicts of interest in the present study.

\section{Ethical consideration}

Plagiarism, consent to publish, misconduct, data fabrication and or falsification, double publication and or submission, and redundancy have been checked by all the authors.

\section{Consent to publish}

Hereby, the authors agreed to publish the article.

\section{Acknowledgments}

The present study was funded by the Vice-Chancellor for Research and Technology of Semnan University of Medical Sciences (ethical code. IR.SEMUMS.REC.1398.162), and the authors would like to express their appreciation.

\section{REFERENCES}

Bennett GJ and Xie YK (1988). A peripheral mononeuropathy in rat that produces disorders of pain sensation like those seen in man. Pain, 33(1): 87107. DOI: https://www.doi.org/10.1016/0304-3959(88)90209-6

Carrasco C, Naziroğlu M, Rodríguez AB, and Pariente JA (2018). Neuropathic pain: Delving into the oxidative origin and the possible implication of transient receptor potential channels. Frontiers in Physiology, 9: 95: 1-15. DOI: https://www.doi.org/10.3389/fphys.2018.00095

Chanchal SK, Mahajan UB, Siddharth S, Reddy N, Goyal SN, Patil PH, Bommanahalli BP, Kundu CN, Patil CR, and Ojha S (2016). In vivo and in vitro protective effects of omeprazole against neuropathic pain. Scientific Reports, 6: 1-10. DOI: https://www.doi.org/10.1038/srep30007

Deng J, Han J, Chen J, Zhang Y, Huang Q, Wang Y, Qi X, Liu Z, Leung EL, Wang D et al. (2021). Comparison of analgesic activities of aconitine in different mice pain models. PLoS One, 16(4): e0249276. DOI: https://www.doi.org/10.1371/journal.pone.0249276

Deuis J R, Dvorakova L S, and Vetter I (2017). Methods used to evaluate pain behaviors in rodents. Frontiers in Molecular Neuroscience, 10 : 1-17. DOI: https://www.doi.org/10.3389/fnmol.2017.00284

Duraku LS, Hossaini M, Hoendervangers S, Falke LL, Kambiz S, Mudera VC, Holstege JC, Walbeehm ET, and Ruigrok TJ (2012). Spatiotemporal dynamics of re-innervation and hyperinnervation patterns by uninjured CGRP fibers in the rat foot sole epidermis after nerve injury. Molecular Pain, 8: 61: 1-13. DOI: https://www.doi.org/10.1186/1744-8069-8-61

Jensen TS, and Finnerup NB (2014). Allodynia and hyperalgesia in neuropathic pain: Clinical manifestations and mechanisms. The Lancet. Neurology, 13(9): 924-935. DOI: https://www.doi.org/10.1016/S1474-4422(14)70102-4

Ji RR, and Suter MR (2007). P38 MAPK, microglial signaling, and neuropathic pain. Molecular Pain, 3: 1-9. DOI: https://www.doi.org/10.1186/1744$\underline{8069-3-33}$ 
Kauppila T, Kontinen VK, and Pertovaara A (1998). Weight bearing of the limb as a confounding factor in assessment of mechanical allodynia in the rat. Pain, 74(1): 55-59. DOI: https://www.doi.org/10.1016/S0304-3959(97)00143-7

Kingery WS (1997). A critical review of controlled clinical trials for peripheral neuropathic pain and complex regional pain syndromes. Pain, 73(2): 123-139. DOI: https://www.doi.org/10.1016/S0304-3959(97)00049-3

Masri R, Quiton RL, Lucas JM, Murray PD, Thompson SM, and Keller A (2009). Zona incerta: A role in central pain. Journal of Neurophysiology, 102(1): 181-191. DOI: https://www.doi.org/10.1152/jn.00152.2009

Minett MS, Quick K, and Wood JN (2011). Behavioral measures of pain thresholds. Current protocols in Mouse Biology, 1(3): 383-412. DOI: https://www.doi.org/10.1002/9780470942390.mo110116

Mizisin AP, Kalichman MW, Garrett RS, and Dines KC (1998). Tactile hyperesthesia, altered epidermal innervation and plantar nerve injury in the hindfeet of rats housed on wire grates. Brain Research, 788: 13-19. DOI: https://www.doi.org/10.1016/S0006-8993(97)01474-1

Mogil JS (2009). Animal models of pain: Progress and challenges. Nature Reviews. Neuroscience 10(4): 283-294. DOI: https://www.doi.org/10.1038/nrn2606

Pitcher GM, Ritchie J, and Henry JL (1999). Paw withdrawal threshold in the von frey hair test is influenced by the surface on which the rat stands. Journal of Neuroscience Methods, 87(2): 185-193. DOI: https://www.doi.org/10.1016/S0165-0270(99)00004-7

Pop NL, Nan A, Urda-Cimpean AE, Florea A, Toma VA, Moldovan R, Decea N, Mitrea DR, and Orasan R (2021). Chitosan functionalized magnetic nanoparticles to provide neural regeneration and recovery after experimental model induced peripheral nerve injury. Biomolecules, 11(5): 1-22. DOI: https://www. doi.org/10.3390/biom11050676

Popiolek-Barczyk K, and Mika J (2016). Targeting the microglial signaling pathways: New insights in the modulation of neuropathic pain. Current Medicinal Chemistry, 23(26): 2908-2928. DOI: https://www.doi.org/10.2174/0929867323666160607120124

Ren K (1999). An improved method for assessing mechanical allodynia in the rat. Physiology and Behavior, 67(5): 711-716. DOI: https://www.doi.org/10.1016/S0031-9384(99)00136-5

Ren K, Thomas DA, and Dubner R (1995). Nerve growth factor alleviates a painful peripheral neuropathy in rats. Brain Research, 699(2): $286-292$. DOI: https://www.doi.org/10.1016/0006-8993(95)00920-L

Safakhah HA, Bazargani A, and Ghanbari A (2016). Effects of forced exercise on neuropathic pain induced by chronic constriction injury of sciatic nerve in male rat. Koomesh, 17(2): 411-418. Available at : http://koomeshjournal.semums.ac.ir/article-1-2969-en.html

Safakhah HA, Moradi Kor N, Bazargani A, Bandegi AR, Gholami Pourbadie H, Khoshkholgh-Sima B, and Ghanbari A (2017). Forced exercise attenuates neuropathic pain in chronic constriction injury of male rat: An investigation of oxidative stress and inflammation. Journal of Pain Research, 10: 1457-1466. DOI: https://www.doi.org/10.2147/JPR.S135081

Santos-Nogueira E, Redondo Castro E, Mancuso R, and Navarro X (2012). Randall-selitto test: A new approach for the detection of neuropathic pain after spinal cord injury. Journal of Neurotrauma, 29(5): 898-904. DOI: https://www.doi.org/10.1089/neu.2010.1700

Soignier RD, Taylor BK, Baiamonte BA, Lee FA, Paul D, and Gould HJ III (2011). Measurement of cfa-induced hyperalgesia and morphine-induced analgesia in rats: Dorsal vs plantar mechanical stimulation of the hindpaw. Pain Medicine (Malden, Mass.), 12(3): 451-458. DOI: https://www.doi.org/10.1111/j.1526-4637.2011.01066.x

Taiwo YO, Coderre TJ, and Levine JD (1989). The contribution of training to sensitivity in the nociceptive paw-withdrawal test. Brain Research, 487(1): 148-151. DOI: https://www.doi.org/10.1016/0006-8993(89)90950-5

Tal M, and Bennett GJ (1994). Extra-territorial pain in rats with a peripheral mononeuropathy: Mechano-hyperalgesia and mechano-allodynia in the territory of an uninjured nerve. Pain, 57(3): 375-382. DOI: https://www.doi.org/10.1016/0304-3959(94)90013-2

Vafaei AA, Safakhah HA, Jafari S, Tavasoli A, Rashidy-Pour A, Ghanbari A, Seyedinia SA, and Tarahomi P (2020). Role of cannabinoid receptors in crocin-induced hypoalgesia in neuropathic pain in rats. Journal of Experimental Pharmacology, 12: 97-106. DOI: https://www.doi.org/10.2147/JEP.S250738

Xiao WH, and Bennett GJ (1994). Magnesium suppresses neuropathic pain responses in rats via a spinal site of action. Brain Research, 666(2): 168172. DOI: https://www.doi.org/10.1016/0006-8993(94)90768-4

Yam MF, Loh YC, Oo CW, and Basir R (2020). Overview of neurological mechanism of pain profile used for animal "pain-like" behavioral study with proposed analgesic pathways. International Journal of Molecular Sciences, 21(12): 1-26. DOI: https://www.doi.org/10.3390/ijms21124355 